\title{
Application Management Practice of Investment Statistics Business Based on Blockchain Technology
}

\author{
Lei $\mathrm{Ma}^{1, *}$, Jiachen $\mathrm{Che}^{2}$, Xiao Qian ${ }^{3}$, Hongfang $\mathrm{Song}^{4}$, Geping Wen ${ }^{5}$, Hao Jiang ${ }^{6}$, Qinyue Cui ${ }^{7}$, Fan Wen ${ }^{8}$ \\ ${ }^{1}$ Development Planning Department, State Grid Zhejiang Electric Power Co., Ltd, Hangzhou, Zhejiang Province \\ ${ }^{2}$ Strategy and development Research Center, State Grid Zhejiang Economic Research Institute, Hangzhou, Zhejiang Province \\ ${ }^{3}$ Development Planning Department, State Grid Zhejiang Electric Power Co., Ltd, Hangzhou, Zhejiang Province \\ ${ }^{4}$ Development Planning Department, State Grid Zhejiang Electric Power Co., Ltd, Hangzhou, Zhejiang Province \\ ${ }^{5}$ Development Planning Department, State Grid Ningbo Electric Power Supply Company, Ningbo, Zhejiang Province \\ ${ }^{6}$ Development Planning Department, State Grid Ningbo Electric Power Supply Company, Ningbo, Zhejiang Province \\ ${ }^{7}$ Development Planning Department, State Grid Ningbo Electric Power Supply Company, Ningbo, Zhejiang Province \\ ${ }^{8}$ Strategy and development Research Center, State Grid Zhejiang Economic Research Institute, Hangzhou, Zhejiang Province
}

\begin{abstract}
As a new decentralized infrastructure and distributed computing paradigm, blockchain is of great significance to break through the "mutual trust problem" between statistical specialty and basic data providing departments, and between the upper and lower levels of statistical data reporting units, and further improve the quality of statistical work. This paper first briefly analyzes the problems existing in the current investment statistics business, and introduces the basic principles and characteristics of blockchain technology. This paper studies the idea and process optimization of the application of blockchain technology in investment statistics business, and expounds the specific application schemes of blockchain technology in different links, such as automatic data collection, automatic data verification, automatic report generation, and automatic index release. Finally, the expected effect of the application of blockchain technology in investment statistics business is analyzed.
\end{abstract}

\section{Problems in current investment statistics}

In the current investment statistics business, there are many problems in the submission of investment statistics report, such as large workload of data manual verification, random report data submission, poor data quality, and many circulation links involved in account data generation, which easily leads to unclear data responsibility ownership. Blockchain is a kind of data structure formed by orderly linking of blocks, in which block refers to the collection of data, and relevant information and records are included, which is the basic unit of forming blockchain. In order to ensure the traceability of the blockchain, each block will be marked with a time stamp as a unique mark. The special data structure organization form of blockchain makes blockchain technology have four main characteristics: decentralization, transparency, contract execution automation and traceability. The decentralization, transparency, fairness and openness of blockchain technology can solve the problems that investment statistics business involves multi department collaboration, multi-level interaction, and the data maintenance requirements of various business departments are uneven, and the investment completion data is disturbed by assessment. At the same time, it can also solve the "mutual trust problem" between the statistical specialty and the basic data providing department, and between the superior and subordinate of the statistical data reporting unit.

\section{Application ideas and process optimization of blockchain Technology}

\subsection{Application ideas of blockchain Technology}

Focusing on the core business of Investment Statistics basic data collection, verification, calculation, audit, confirmation and release of fixed assets investment statistics report, the investment statistics application based on blockchain technology is carried out, and the construction department, equipment department, finance department, development department and other departments at all levels, project management units, construction, supervision and owner three major project departments involved in the report business are carried out Connected as the main body of the blockchain. The smart contract elements such as basic data verification rules, investment collection value algorithm, report automatic verification rules and other intelligent contract elements

\footnotetext{
*Corresponding author: malei@zj.sgcc.com.cn
} 
are clarified, and a typical application of automatic investment statistics blockchain integrating automatic source collection, data automatic verification, report automatic generation and index automatic publishing is established. By changing the traditional statistical business model, we can improve the authenticity, accuracy, intelligence and synergy of investment statistics business data.

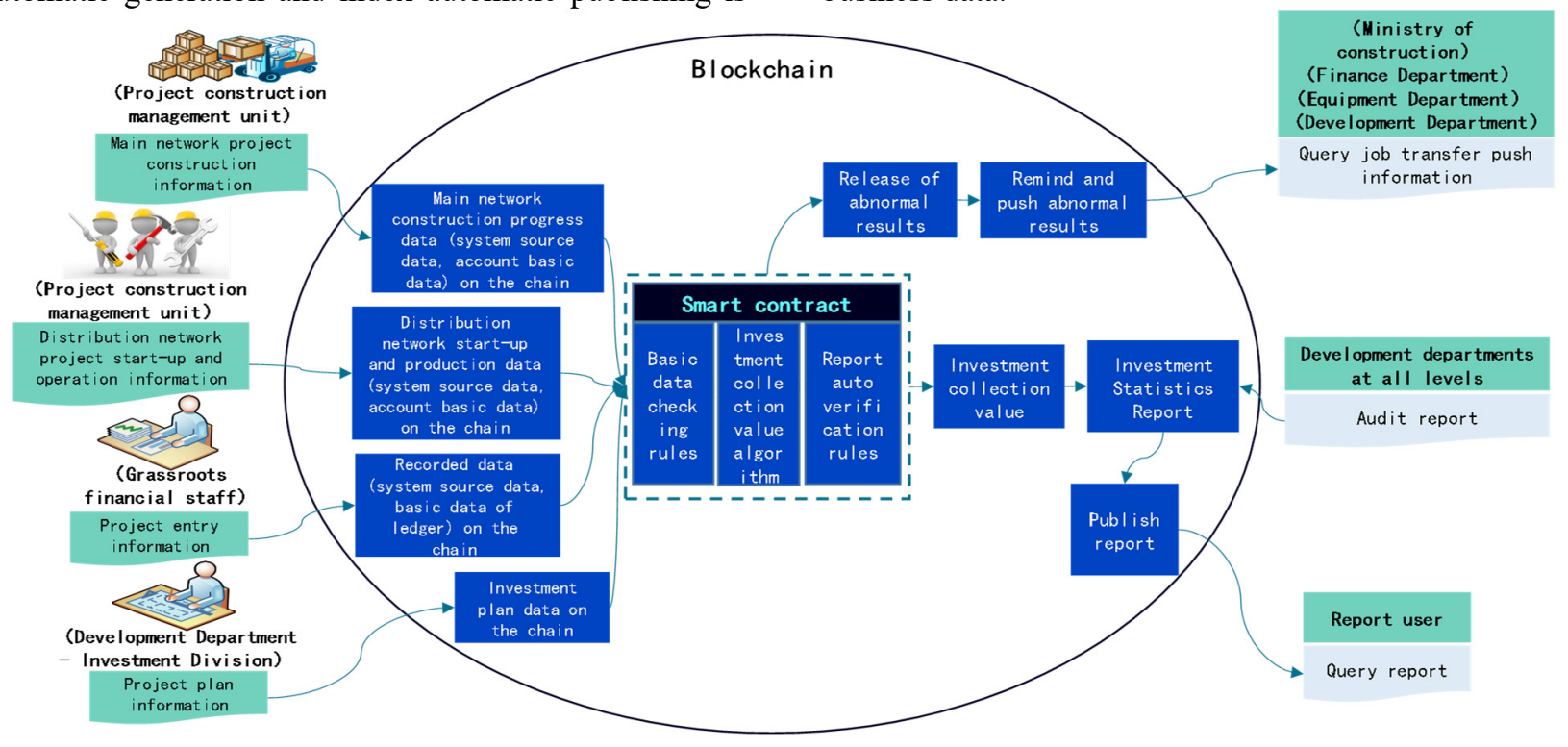

Figure 1. Main ideas for investment statistics application based on blockchain technology

\subsection{Business optimization process of investment statistics report based on "blockchain + algorithm"}

Integrate the "block chain + algorithm", combine the characteristics of investment statistics business, optimize the business process of investment statistics report, and realize the automatic statistics of the whole process of investment statistics report. In the stage of basic data acquisition, the link data is defined. Once the data is linked, the online automatic verification of basic data, manual verification of key data, confirmation of basic data, automatic calculation of investment completion, automatic verification of report, audit (confirmation) of report and release of report are all completed on the chain. The specific steps are as follows:

- 2.2.1 Basic data collection (submission). Basic data collection (submission stage) mainly clarifies which data is on the chain, the purpose of the data on the chain, the source of the data, and the maintenance personnel, departments, systems, maintenance times, and versions of each data information.

- 2.2.2 The basic data is checked automatically on the Internet. The online automatic verification rules of basic data are part of the smart contract, including the following two aspects:

- 2.2.2.1 the consistency comparison between the source data automatically collected by the system and the basic data submitted to the account.

- 2.2.2.2 the automatic verification of the basic data.
- 2.2.3 Key data are checked manually. For the items that pass the second step of online automatic verification of basic data and manual verification of white list, manual verification should be conducted again, including whether the basic data of key milestone nodes is consistent with the actual situation on site.

- 2.2.4 Basic data confirmation. Through the second step of online automatic verification and the third step of manual verification of basic data, professional departments (infrastructure, finance, development, equipment and other departments) corresponding to the basic data need to confirm the data.

- 2.2.5 The investment is calculated automatically. Using the data confirmed by all professional departments in step 4, the system automatically calculates and generates the investment collected value based on the calculation method of investment collection value (part of smart contract).

- 2.2.6 The report is checked automatically. According to the investment collection value automatically calculated by the system in the fifth step, the automatic verification rules of investment statistical report (part of smart contract) are set to automatically check the generated investment statistical report.

- 2.2.7 Report review (confirmation). Through the sixth step report, the submitted investment statistical report is checked automatically by the auditor (the same level unit). The report auditor will submit the approved 
report to the relevant personnel of the higher level unit (different level units, such as headquarters) for audit. The approved report can generate investment statistics account synchronously.

- 2.2.8 Report publishing. After the report is approved in step 7, the approved report can be released. Under the condition that the demand of each report user is clear, the investment statistical report is uniformly issued by the statistics professionals for the report users to query and use.

\section{Specific application plan of investment statistics business based on blockchain technology}

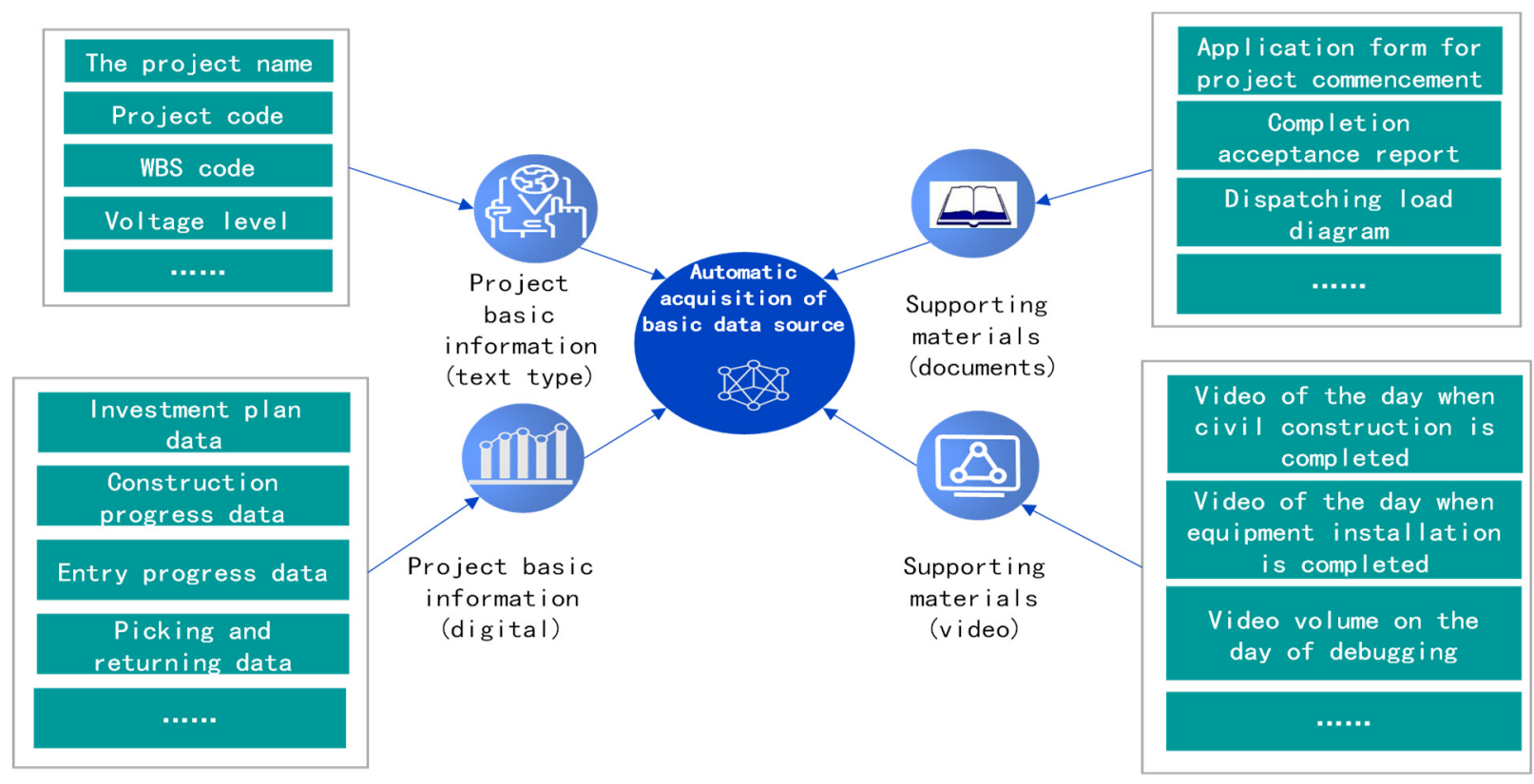

Figure 2. Investment statistics blockchain data pool

\subsection{The intelligent contract is executed automatically, and the data is checked and calculated automatically}

Through smart contract, business verification rules and algorithms such as basic data verification rules, investment collection value algorithm, deviation warning

\subsection{Automatic collection of basic data source, construction of investment statistics blockchain data pool}

Collect the source side structured data such as project budget estimate, construction scale, construction progress, cost entry and investment plan in the source end systems such as planning plan, infrastructure management and control system, ERP, PMS, as well as unstructured data such as main milestone node progress supporting materials in the form of videos, images and documents collected by intelligent terminals to build the investment statistics blockchain data pool.

and other business verification rules and algorithms can be transformed into contracts automatically executed on the blockchain platform. Once the conditions for contract execution are met, automatic verification and report value automatic calculation can be started. The execution results of smart contracts can also be publicly checked on the blockchain to simultaneously improve the fairness and transparency of contracts. 
(1) Basic data checking rules

a Verification rules of milestone chain

- Verification rules of investment control chain:

- Verification rules of capital chain

- Verification rules of logistics chain;

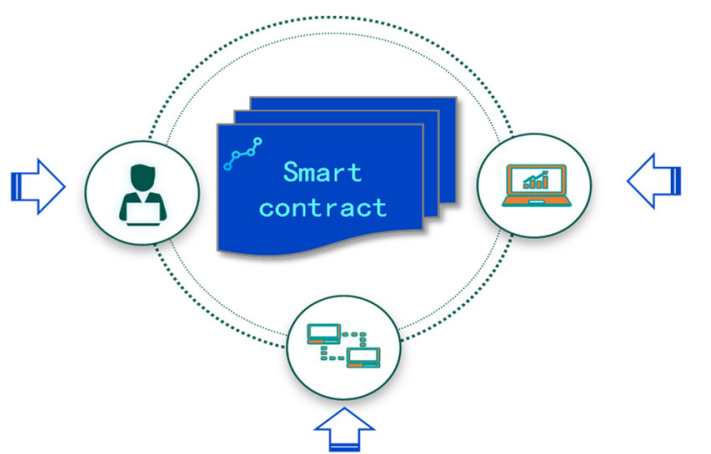

(3) Business rules and algorithms for automatic verification of reports such as deviation warning

- Deviation between investment progress and construction progress;

- Deviation between construction progress and recorded progress:

Deviation between construction

progress and recorded progress:
(2) Investment collection value algorithm

- Algorithm of investment acquisition

value for $35 \mathrm{kV}$ and above power

grid infrastructure projects;

Algorithm of investment acquisition

value for $10 \mathrm{kV}$ and below powe

grid infrastructure projects;

Figure 3. Smart contract rules

3.2.1 Basic data checking rules. Based on the actual management requirements and business rules, this paper combs the source side data verification rules from two aspects of time logic and scale consistency, so as to realize the automatic verification function of basic data in multiple dimensions, such as balance relationship, logical relationship, comparison relationship and cohesion relationship. It mainly includes verifying the accuracy and rationality of the data fields involved in the production capacity report and investment completion report. The automatic checking rules of basic data are hard rules (must pass, otherwise it can't be confirmed to flow to the next link).

3.2.2 Investment collection value algorithm. In view of the current situation of "manual submission and inconsistent standards" in investment statistics of traditional power grid infrastructure projects, combined with the reporting requirements of the National Bureau of statistics for fixed assets investment, the calculation method for investment completion of power grid infrastructure projects of the company is unified, and the system access source is defined. The algorithm models of investment collection value of power grid infrastructure projects with $35 \mathrm{kV}$ and above, $10 \mathrm{kV}$ and below are constructed respectively to realize the investment statement value automatic calculation. Based on the calculation method of investment collection value, the system automatically calculates and generates the investment collection value based on the data confirmed by each professional department. The specific calculation method of investment collection value of each voltage level is as follows:

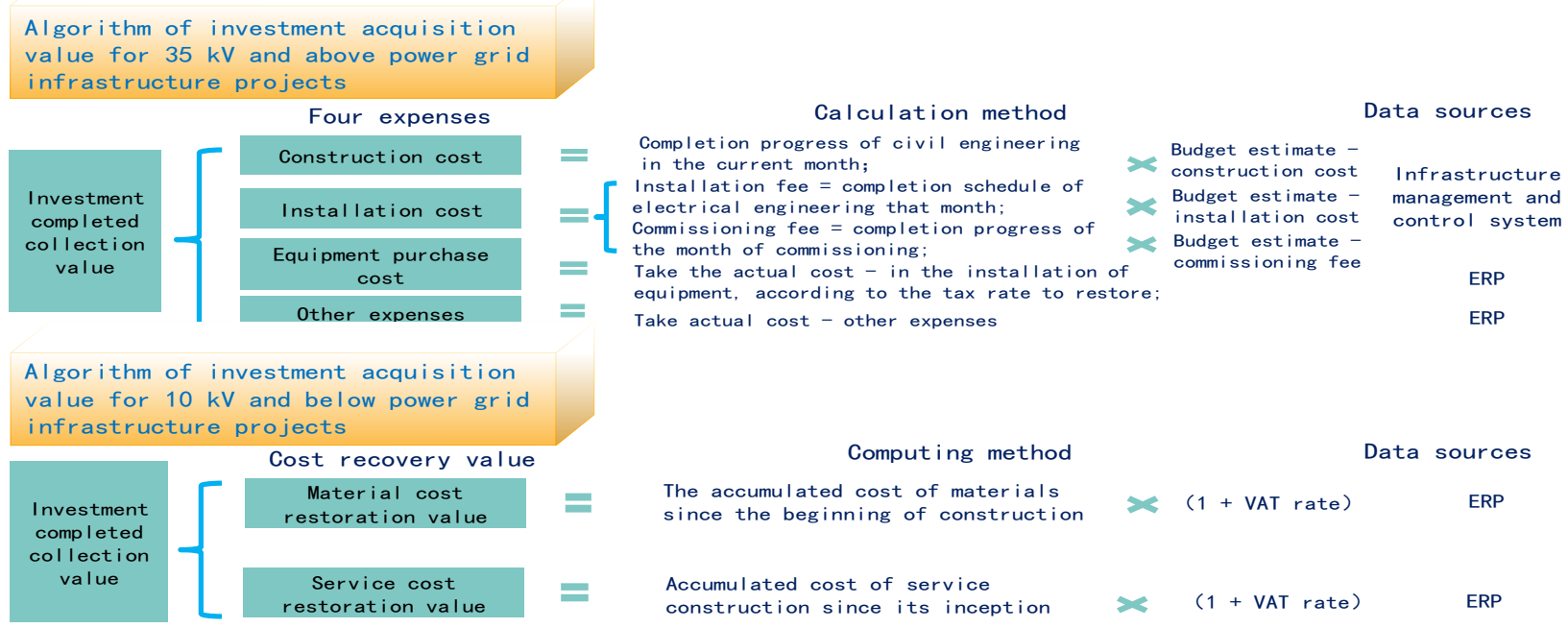

Figure 4. Calculation method of investment collection value

3.2.3 Basic data checking rules. Based on the actual management requirements and business rules, this paper combs the source side data verification rules from two aspects of time logic and scale consistency, so as to realize 
the automatic verification function of basic data in multiple dimensions, such as balance relationship, logical relationship, comparison relationship and cohesion relationship. It mainly includes verifying the accuracy and rationality of the data fields involved in the production capacity report and investment completion report. The automatic checking rules of basic data are hard rules (must pass, otherwise it can't be confirmed to flow to the next link).

\subsection{The audit process is online and traceable}

The investment statistics report business based on blockchain technology can realize all online operations of investment statistics data collection, verification, calculation, audit, confirmation and other processes, improve the level of automation, reduce the degree of manual intervention, and improve the efficiency of statistical work; At the same time, the key basic data, supporting material information, automatic verification results, audit or confirmation status, report value and relevant maintenance personnel, time stamp, version and other information involved in each project are recorded into the data block one by one, so as to realize the trace and traceability of investment statistical report data.

\subsection{Report is automatically published and shared by data sharing}

The application of blockchain technology in investment statistics business realizes the automatic release of reports and the sharing of data among all participants in the blockchain.

\section{Expected application effect}

Change the management model and promote true and transparent statistics. Innovative use of "blockchain $+\mathrm{X}$ " investment statistics business applications, relying on new technologies such as smart terminal collection, blockchain, etc., through the development of data verification rules, unified report calculation methods, deviation warnings and other AI algorithm applications to transform statistical business management mode. Automatically collect data and clarify data responsibilities from the source; automatic data verification and move forward to ensure data reliability from the process; achieve data transparency throughout the process, and promote the sharing of management data among various disciplines.

Landing automatic statistics, improve the efficiency of statistical work. By using the blockchain technology and the automatic trigger and execution mode of smart contract, the whole business process of investment statistics is online, and the statistical work efficiency is improved automatically; At the same time, it automatically connects, interacts and cooperates with various business departments to realize the digitization, intellectualization and automatic closed-loop of investment statistics business, and effectively implements the "online power grid" automatic statistics.

Rebuild the trust model and improve the data quality in an all-round way. Relying on the blockchain consensus mechanism to solve the "mutual trust problem" has changed the drawbacks of the traditional mode of data "easy to tamper with and opaque". All operations and relevant maintenance personnel information are included in the blockchain, which ensures the traceability of all operations, clarifies the responsible party of data, ensures the authenticity and credibility of data, and comprehensively improves the quality of statistical data.

\section{Conclusion}

As a new database technology, blockchain technology has the characteristics of decentralization, openness and transparency, which is a "sharp weapon" to solve the trust problem. The introduction of blockchain technology into investment statistics business will bring changes to the traditional investment statistics management mode. In the future, we should fully consider the impact of the application of blockchain technology on investment statistics management process, formulate a new version of investment statistics management system based on blockchain technology, clarify the business process and work responsibilities of various professional departments, and institutionalize and standardize them. The project progress account is directly generated by the construction front line of the project, and the new version of investment statistical report data standard based on blockchain technology is formulated to clarify the data source, data verification standard and other contents, so as to make the collection, verification, generation, circulation, use and sharing of investment statistical report data have rules to follow.

\section{References}

1. Li. W, "Thinking on the application of blockchain technology in statistical survey," China Information, 2020 .

2. Li. X, Wang. H. J, "Power data sharing mechanism based on blockchain," Collection of papers on the annual meeting of power industry informatization, 2019.

3. Yan. Y, Zhou. Z. Q, Tu. Y, Liu. Z. B, Hu. S. S, "Research on Application of Electric Power Data Preservation Based on Blockchain," Power big data, 2019.

4. Chen. X. N, Jiang. Y. G, Jia. L. F, Zhou. J, "Research on blockchain smart contract technology for big data fusion and sharing," Information recording materials, 2019.

5. Zhou. H. Y, Qian. W. H, Bai. J. J, Wei. Z. N, Sun. G. Q, Zang. H. X, "Typical application scenario analysis and project practice of energy blockchain," Power Construction, 2020. 